\title{
The association between benzodiazepine use and sleep quality in residential aged care facilities: a cross-sectional study
}

\author{
Lynna Chen ${ }^{1}$, J. Simon Bell ${ }^{2,3,4}$, Renuka Visvanathan ${ }^{5,6}$, Sarah N. Hilmer ${ }^{3,7}$, Tina Emery ${ }^{8}$, Leonie Robson ${ }^{8}$, \\ Jessica M. Hughes ${ }^{2}$ and Edwin C. K. Tan ${ }^{2,9^{*}}$
}

\begin{abstract}
Background: Benzodiazepines are commonly prescribed in residential aged care facilities (RACFs) for their sedative and anxiolytic effects. The objective of this study was to investigate the association between benzodiazepine use and sleep quality in residents of RACFs.

Methods: A cross-sectional study involving 383 participants was conducted in six Australian RACFs. Night-time sleep quality, day-time drowsiness and day-time napping behavior were assessed using a validated questionnaire. Logistic regression was used to compute adjusted odds ratios (AORs) and 95\% confidence intervals (Cls) for the association between benzodiazepine use and sleep quality. Covariates included pain, dementia severity, depression, insomnia and other sedative use.

Results: Of the 383 residents (mean age 87.5 years, 77.5\% female), 96(25.1\%) used a benzodiazepine on a regular basis. Residents who used long-acting benzodiazepines on a regular basis had higher night-time sleep quality than non-users ( $A O R=4.00,95 \% \mathrm{Cl} 1.06-15.15)$. Residents who used short-acting benzodiazepines on a PRN only basis had longer daytime napping times than non-users ( $\mathrm{AOR}=1.77,95 \% \mathrm{Cl} 1.01-3.08)$. No benzodiazepine category was associated with day-time drowsiness.

Conclusions: The association between benzodiazepine use and sleep quality is dependent on the half-life and prescribing pattern of the benzodiazepine. Short-acting PRN benzodiazepines were associated with lower night time sleep quality and longer day-time napping compared to long-acting regular benzodiazepines. Longitudinal studies are needed to determine whether these findings reflect channeling of short-acting agents to residents at higher risk of sleep disorders.
\end{abstract}

Keywords: Sleep, Sleep disorders, Sleep quality, Benzodiazepines, Hypnotics and sedatives, Homes for the aged, Nursing homes

\section{Background}

Ageing is associated with a decrease in sleep length and quality [1]. This is largely attributed to altered circadian rhythms, lowered melatonin levels and increasing rates of medical co-morbidities. Sleep disturbances manifest

\footnotetext{
* Correspondence: edwin.tan@monash.edu

${ }^{2}$ Centre for Medicine Use and Safety, Faculty of Pharmacy and

Pharmaceutical Sciences, Monash University, 381 Royal Parade, Melbourne, Parkville Victoria 3052, Australia

${ }^{9}$ Aging Research Center, Center for Alzheimer Research, Department of

Neurobiology, Care Sciences and Society, Karolinska Institutet and Stockholm

University, Stockholm, Sweden

Full list of author information is available at the end of the article
}

as decreased total nocturnal sleep time, delayed sleep onset, advanced circadian phase, reduced rapid-eyemovement (REM) sleep and day-time napping and somnolence [2]. Sleep disorders predispose individuals to hypertension, depression, cardiovascular and cerebrovascular disease which further diminish quality of life [3]. In the short term, individuals experience impaired cognition and poor day-to-day functioning. Studies estimate that $50 \%$ of individuals over 60 years of age suffer from insomnia, with a higher prevalence amongst women [4].

Residents of RACFs experience a higher prevalence of sleep disorders. This is likely attributable to environmental 
variables: noise, lighting, temperature and nursing times. It is also attributable to resident characteristics [5]. More than half of residents residing in Australian RACFs have a diagnosis of dementia [6]. People with dementia have higher rates and increased severity of sleep disorders with sleep disturbance present in up to $71 \%$ of people with dementia compared to $55.7 \%$ of people without dementia [7]. Pain is associated with sleep disorders and is also highly prevalent among residents [8].

The management of sleep problems in residents of RACFs is often multifaceted. Non-pharmacological therapies may include exercise, bright light exposure, nighttime continence care, and cognitive behavioral interventions [9]. However, the results of these interventions are often inconsistent or provide modest benefits on selected aspects of sleep quality. The most common treatment for sleep problems is pharmacological. Benzodiazepines, which enhance the inhibitory effect of gamma-aminobutyric acid (GABA), are commonly prescribed for their sedative and anxiolytic properties [3]. Their sleep-promoting effects make them among the most prevalent medication class among older people [10]. Studies have reported the prevalence of regular benzodiazepine use in Australian RACFs ranging from 12 to $42 \%$ [11].

Benzodiazepines are associated with modest improvements to sleep latency and longer sleep duration; however, they suppress deep sleep which compromises sleep's restorative effects [12]. A meta-analysis by Glass et al. found that the benefits associated with sedative use are marginal and are outweighed by the risk of adverse events. This is particularly clinically relevant for patients at a high risk for falls or cognitive impairment [13]. Older people have increased sensitivity to adverse events, despite growing tolerance to the sedative effects [12]. Adverse events include daytime drowsiness, lethargy, fatigue, agitation, memory loss, impaired coordination and falls [14]. Benzodiazepines may also contribute to cognitive decline in people with dementia [15].

A recent Cochrane review revealed a paucity of appropriately conducted studies that have investigated the benefits and risks of benzodiazepines in people with dementia [16]. As low sleep quality is associated with impaired quality of life [17], it is important to study the effects of benzodiazepine use in vulnerable populations such as people with dementia and those living in RACFs.

The objective of this study was to investigate the association between benzodiazepine use and sleep quality in residents of RACFs.

\section{Methods}

\section{Design and setting}

Data were drawn from a cross-sectional study of permanent residents in six low-level and high-level RACFs in metropolitan Adelaide (population 1.2 million) and regional Mt Gambier (population 25,000), South Australia. In Australia, RACFs predominantly provide support and service to older individuals unable to live independently at home due to frailty, disability or illness. RACFs were classified according to level of support: ranging from aided daily tasks and personal care in low-level RACFs to 24-h nursing in high-level RACFs. The full study protocol has been described previously [18].

\section{Sample}

Permanent residents aged 65 years or older were invited to participate in the study. Inclusion criteria were ability to participate in structured assessments in English. Residents deemed medically unstable (e.g. delirium) or estimated to have less than three months to live by facility staff were excluded from participation.

Of the 664 eligible residents in six RACFs, 603 were invited to participate. Of the 603 residents invited to participate, 220 were excluded (106 declined; 34 were unwell, hospitalized or palliative; 54 had a third party who could not be contacted or did not provide consent; and 26 were excluded for other reasons). The final sample comprised 383 residents. Participants were similar to all residents of the RACFs in terms of age (87.5 years [SD 6.2] vs. 87.3 years [SD 6.4], $p=0.66)$, sex ( $77.5 \%$ female vs. $78.5 \%$ female, $p=0.90$ ) and dementia diagnosis (44.1\% vs. $46.8 \%, p=0.72)$.

\section{Data source}

All data were collected by three experienced study nurses who underwent centralized training in the standard administration of the study assessment tools. Data collection took place between April and August 2014.

Demographic, diagnostic and medication data were extracted from each resident's electronic medical record and medication chart. A standard data extraction form, comprising a series of validated and widely used data collection scales, enabled collection of other clinical data. Both resident self-report and observational scales completed by staff informants were utilized [18]. Where possible, residents chose the most appropriate response through verbal or written communication. If residents were unable, a staff informant with at least two weeks familiarity with the specified resident completed the scale.

\section{Medication assessment}

Data on all charted medications were extracted from each resident's medication chart. Regular and as-needed (PRN) prescription, non-prescription and complementary and alternative medications were considered. Regular use was defined as a documented regular sequence of administration in the resident's chart.

Medications were coded according to the Anatomical Therapeutic Chemical (ATC) classification system, as 
recommended by the World Health Organization. Benzodiazepines were defined as anxiolytic benzodiazepine derivatives (N05BA), hypnotic and sedative benzodiazepine derivatives (N05CD) or benzodiazepine related drugs (N05CF). A resident was considered a benzodiazepine user if he or she was charted a benzodiazepine on a regular or PRN basis. Benzodiazepines were classified according to half-life into two categories: short-acting (half-life $\leq 24 \mathrm{~h}$ ) and long-acting (half-life $>24 \mathrm{~h}$ ). Temazepam, oxazepam, alprazolam, bromazepam, triazolam, midazolam, lorazepam, zopiclone and zolpidem were classified as short-acting benzodiazepines. Diazepam, clobazam, nitrazepam, flunitrazepam were classified as long-acting benzodiazepines. Non-benzodiazepine sedative drugs included other sedatives and hypnotics (e.g. melatonin, antihistamines) and medications with prominent sedative side effects (e.g. antipsychotics, antidepressants, antiepileptics, opioid analgesics).

\section{Sleep quality}

The primary outcome measure was sleep quality assessed using a previously validated six-item questionnaire [19]. The questionnaire comprised three domains: night-time sleep quality (overall self-reported sleep quality, sleeping duration, restfulness), day-time drowsiness and day-time napping behavior (frequency and duration) (Table 2). The six-item questionnaire has been used previously among older people living in self- and assisted-care villages to assess the association between sleep quality and falls [20].

The scoring of the individual sleep domains within the questionnaire was adapted from the method described by St George et al [20]. The night-time sleep quality score was derived from an aggregate of the three nighttime sleep items, with equal weighting for each domain. This yielded a night-time sleep quality score ranging between 3 and 15 . Higher night-time sleep quality was operationally defined as a score of 3-10 and lower sleep quality as a score of 11-15, based on median score. Daytime drowsiness was dichotomized as drowsy or nondrowsy. Average number of hours napping per week was calculated from the questions pertaining to napping frequency and duration. Residents were categorized as napping $<3.5 \mathrm{~h}$ per week (less than half an hour per day), or $\geq 3.5 \mathrm{~h}$ per week [20].

\section{Covariates}

Co-morbidities were extracted from resident electronic medical records and included depression, insomnia and history of falls. The Charlson Comorbidity Index (CCI) was calculated for each resident [21]. Dementia severity was measured in residents with and without a dementia diagnosis with the Dementia Severity Rating Scale [22]. Resident agitation and aggression was assessed using the Neuropsychiatric Inventory Nursing Home version (NPI-
$\mathrm{NH}$ ) [23]. Activities of daily living were assessed with the Katz Activities of Daily Living (ADL) index [24]. Pain was observed and assessed using the Pain Assessment in Advanced Dementia (PAINAD) Scale [25].

\section{Statistical analysis}

Descriptive analyses were conducted using Pearson Chisquare tests for categorical variables, Student's t-tests for continuous variables and Mann-Whitney $U$ tests for ordinal variables. Odds ratios (ORs) and 95\% confidence intervals (CIs) of the associations between different benzodiazepine categories and sleep domains were computed with binary logistic regression analyses. Analyses were adjusted for age, sex, dementia severity rating scale, any pain reported on the PAINAD scale, depression, insomnia and nonbenzodiazepine sedative use. These factors were used to adjust the analyses because they were deemed clinically relevant based on previous research [2]. Sensitivity analysis was performed by initially removing insomnia diagnosis to assess for potential over-correction, and this did not affect the results. Benzodiazepine use was analyzed separately based on half-life classification (short or long-acting) and type of prescription (regular or PRN). Separate models were run for the association between each benzodiazepine category and each different sleep quality domain i.e. nighttime sleep quality, day-time drowsiness and day-time napping behavior. The reference groups were those residents not taking these drugs.

Missing values were imputed with multiple imputation with five iterations. This was because multiple imputation is superior to complete case analyses when data are missing at random [26]. Values were imputed for sleep quality questionnaire (24.8\% missing) and PAINAD (1.0\% missing). Analyses were undertaken using the Statistical Package for the Social Sciences (SPSS, version 21.0, Chicago, IL, USA).

\section{Ethical considerations}

The study was approved by the Royal Australian College of General Practitioners (RACGP) National Research and Evaluation Ethics Committee and the Monash University Human Research Ethics Committee. All participants were informed about the study verbally and in writing. Written informed consent was obtained from the residents, or their guardian, next of kin, or significant other if unable. All study procedures were conducted in accordance with the Australian National Statement for Ethical Conduct in Human Research.

\section{Results}

Of the residents, 229 (59.8\%) were able to self-complete the sleep questionnaire, with the remainder completed by a staff informant or carer. Demographic characteristics of residents in RACFs with lower and higher night- 
time sleep quality were similar (Table 1 ). The mean age of residents was 87.5 years $(\mathrm{SD}=6.19)$. Of the 383 participants, $297(77.5 \%)$ were female and 135 (35.2\%) lived in high-level RACFs. In total, 169 (44.1\%) had a dementia diagnosis and 230 (60.1\%) had a depression diagnosis. Residents with lower night-time sleep quality were more likely to exhibit pain ( $34.6 \%$ vs $25.0 \%, p=0.04)$ compared to those with higher night-time sleep quality (Table 1).

Overall, 96 (25.1\%) residents were charted regular benzodiazepines and 121 (31.6\%) were charted PRN benzodiazepines. Eighty residents (20.9\%) used benzodiazepines on a PRN only basis, with the majority being short-acting formulations $(n=69)$. Forty-one $(10.7 \%)$ residents used more than one category of benzodiazepine.

Residents who used benzodiazepines had a higher prevalence of diagnosed insomnia $(21.3 \%$ vs $4.9 \%, p<0.01)$, anxiety ( $44.9 \%$ vs $32.2 \%, p=0.01)$ and depression $(68.0 \%$ vs $53.2 \%, p<0.01)$ than residents who did not use benzodiazepines. Residents taking benzodiazepines had higher prevalence of agitation or aggression compared to those residents not taking benzodiazepines ( $34.3 \%$ vs $19.5 \%, p<0.01$ ).

Resident responses to the six item sleep questionnaire are shown in Table 2 . There were no statistically significant differences between users and non-users of benzodiazepines in any of the sleep questionnaire items or domains.

\section{Night-time sleep quality}

Overall, 181 (47.3\%) residents had lower night-time sleep quality (score $\leq 10$ ). In adjusted analyses, use of regular long-acting benzodiazepines was significantly associated with higher sleep quality $(\mathrm{AOR}=4.00,95 \% \mathrm{CI}=1.06$ 15.15) (Table 3). Use of PRN short-acting benzodiazepines was associated with lower night-time sleep quality, however this did not reach statistical significance $(\mathrm{AOR}=0.59$, $95 \% \mathrm{CI}=0.34-1.02$ ).

\section{Day-time drowsiness}

Of benzodiazepine users, $49.5 \%$ reported day-time drowsiness compared to $55.3 \%$ of non-users. No statistically significant associations were found in unadjusted and adjusted analyses between use of any benzodiazepine category and day-time drowsiness (Table 3).

\section{Average weekly napping time}

There was no significant difference in mean daytime napping time between benzodiazepine users and nonusers overall (3.03 vs $3.01 \mathrm{~h}$ per week, $p=0.97$ ) (Table 1 ). However, the use of PRN only benzodiazepines was found to be associated with increased day-time napping time $(\mathrm{AOR}=1.77,95 \% \mathrm{CI}=1.01-3.08)$.

Table 1 Characteristics of residents with higher and lower sleep quality in Australian residential aged care facilities

\begin{tabular}{|c|c|c|c|c|c|c|}
\hline & \multirow{2}{*}{$\begin{array}{l}\text { All residents } \\
383\end{array}$} & \multicolumn{2}{|c|}{ Higher sleep quality (NTSQS >10) } & \multicolumn{2}{|c|}{ Lower sleep quality (NTSQS $\leq 10)$} & \multirow[t]{2}{*}{$P$ value } \\
\hline & & 202 & $53 \%$ & 181 & $47 \%$ & \\
\hline \multicolumn{7}{|l|}{ Demographic characteristics } \\
\hline Age (mean $\pm \mathrm{SD})$ & $87.53(6.19)$ & 87.62 & $(6.16)$ & 87.43 & $(6.22)$ & $0.77^{\mathrm{a}}$ \\
\hline Female & 297 & 160 & $79.2 \%$ & 137 & $75.7 \%$ & $0.39^{b}$ \\
\hline High Level Care & 135 & 79 & $39.1 \%$ & 56 & $30.9 \%$ & $0.09^{b}$ \\
\hline Born in Australia & 298 & 150 & $74.3 \%$ & 148 & $81.8 \%$ & $0.10^{\mathrm{b}}$ \\
\hline \multicolumn{7}{|l|}{ Functional characteristics } \\
\hline Charlson's comorbidity index (median, IQR) & $2.00(1.00,3.00)$ & 2.00 & $(1.00,3.00)$ & 2.00 & $(1.00,4.00)$ & $0.39^{c}$ \\
\hline ADLs (median, IQR) & $1.00(4.00,6.00)$ & 3.00 & $(1.00,6.00)$ & 4.00 & $(1.00,6.00)$ & $0.66^{c}$ \\
\hline DSRS (mean \pm SD) & 19.09 (16.87) & 20.19 & $(16.57)$ & 17.85 & $(17.10)$ & $0.21^{\mathrm{a}}$ \\
\hline PAINAD, any & 113 & 50 & $24.8 \%$ & 63 & $34.8 \%$ & $0.04^{\mathrm{b}}$ \\
\hline \multicolumn{7}{|l|}{ Medical diagnoses and medications } \\
\hline Dementia & 169 & 94 & $46.5 \%$ & 75 & $41.4 \%$ & $0.35^{\mathrm{b}}$ \\
\hline Insomnia & 48 & 22 & $10.9 \%$ & 26 & $14.4 \%$ & $0.37^{\mathrm{b}}$ \\
\hline Anxiety & 146 & 78 & $38.6 \%$ & 68 & $37.6 \%$ & $0.81^{\mathrm{b}}$ \\
\hline Depression & 230 & 115 & $56.9 \%$ & 115 & $63.5 \%$ & $0.21^{\mathrm{b}}$ \\
\hline Any non-benzodiazepine sedatives & 272 & 145 & $71.8 \%$ & 127 & $70.2 \%$ & $0.71^{b}$ \\
\hline
\end{tabular}

NTSQS night-time sleep quality score, $A C A T$ aged care assessment team, ADLs activities of daily living, DSRS dementia severity rating scale, PAINAD Pain Assessment in Advanced Dementia Scale. SD standard deviation, IQR interquartile range

a.Student's $t$-test

b.Pearson's Chi-square test

c.Mann-Whitney $U$ test 
Table 2 Sleep quality in users and non-users of benzodiazepines in Australian residential aged care facilities. (Based on 6-item questionnaire and derived sleep domain scores)

All residents $(n=383) \quad$ Non-Benzodiazepine user $(n=204) \quad$ Benzodiazepine user $(n=179) \quad P \quad$

Q1: SLEEP QUALITY: Over the past month rate the quality of your night time sleep?

$\begin{array}{llllll}\text { Very poor } & 10 & 3 & 1.5 \% & 7 & 3.9 \% \\ \text { Poor } & 44 & 19 & 9.3 \% & 25 & 14.0 \% \\ \text { Okay } & 96 & 56 & 27.5 \% & 40 & 22.3 \% \\ \text { Good } & 162 & 84 & 41.2 \% & 78 & 43.6 \% \\ \text { Very good } & 72 & 43 & 21.1 \% & 29 & 16.2 \%\end{array}$

Q2: SLEEP DURATION: How much sleep

do you get each night?

$\begin{array}{ll}<4 h & 7 \\ 4-6 h & 89 \\ 6-8 h & 180 \\ 6-10 h & 84 \\ >10 h & 22\end{array}$

Q3: RESTFUL SLEEP: How restless or restful is your sleep as a rule?

$\begin{array}{ll}\text { Very restless } & 10 \\ \text { Restless } & 62 \\ \text { Not very } & 60 \\ \text { Restful } & 191\end{array}$

Very restful

Q4: WAKING CONDITION: How clear headed do you feel when you wake in the morning?

$\begin{array}{ll}\text { Alert } & 184 \\ \text { Slightly drowsy } & 151 \\ \text { Fairly drowsy } & 33 \\ \text { Very drowsy } & 15\end{array}$

Q5: NAPPING FREQUENCY: How regularly do you sleep, nap, or lie down during the day?

Nearly every day

A few times a week

Once or twice a week

Once or twice a month

Never or hardly ever

Q6: NAPPING DURATION: In general how much sleep would you say you get on these days?

None

Less than $1 / 2 \mathrm{~h}$

$1 / 2$ to $1 \mathrm{~h}$

1 to $2 \mathrm{~h}$

More than $2 \mathrm{~h}$

7
89
180
84
22

$1.5 \%$

$21.1 \%$

$45.6 \%$

$24.0 \%$

$7.4 \%$

10

2

60

60

$42.6 \%$

$14.7 \%$

$10.3 \%$

$10.3 \%$

$22.1 \%$

11

7

$3.9 \%$

\section{$-$}


Table 2 Sleep quality in users and non-users of benzodiazepines in Australian residential aged care facilities. (Based on 6-item questionnaire and derived sleep domain scores) (Continued)

\begin{tabular}{|c|c|c|c|c|c|c|}
\hline \multicolumn{7}{|l|}{ SLEEP QUALITY DOMAINS } \\
\hline \multicolumn{7}{|l|}{ Night-time Sleep Quality Score (Category) } \\
\hline Higher & 202 & 113 & $55.4 \%$ & 89 & $49.7 \%$ & \multirow[t]{2}{*}{$0.26^{a}$} \\
\hline Lower & 181 & 91 & $44.4 \%$ & 90 & $50.6 \%$ & \\
\hline Night-time Sleep Quality Score (Mean) & $10.29(2.45)$ & 10.52 & $(2.37)$ & 10.02 & $(2.52)$ & $0.05^{\mathrm{b}}$ \\
\hline \multicolumn{7}{|l|}{ Day-time Drowsiness (Category) } \\
\hline Alert & 183 & 103 & $50.5 \%$ & 80 & $44.7 \%$ & \multirow[t]{2}{*}{$0.26^{c}$} \\
\hline Drowsy & 200 & 101 & $49.5 \%$ & 99 & $55.3 \%$ & \\
\hline \multicolumn{7}{|l|}{ Napping Score (Category) } \\
\hline$<3.5 \mathrm{~h} /$ week & 256 & 137 & $67.2 \%$ & 119 & $66.5 \%$ & \multirow[t]{2}{*}{$0.82^{a}$} \\
\hline$\geq 3.5 \mathrm{~h} /$ week & 127 & 67 & $32.8 \%$ & 60 & $33.5 \%$ & \\
\hline Napping Score (Mean) & 3.02 & 3.01 & & 3.03 & & $0.97^{\mathrm{b}}$ \\
\hline
\end{tabular}

a.Mann-Whitney $\mathrm{U}$ test

b.Student's $t$-test

c.Pearson's chi-square tes

\section{Discussion}

This was one of the first studies to investigate the association between benzodiazepines and sleep quality in RACFs $[27,28]$. The primary finding was that PRN only shortacting benzodiazepine use was associated with lower nighttime sleep quality while regular long-acting benzodiazepine use was associated with higher night-time sleep quality.

The prevalence of benzodiazepine use amongst RACF residents in our study was similar to previous Australian and international studies $[11,29,30]$. The proportion of residents classified as having lower night-time sleep quality is similar to previous research that reports between $40-65 \%$ of older adults have some form of sleep disturbance [31]. St George et al., using the same sixitem questionnaire, found a similar prevalence of lower night-time sleep quality and an association between pain and lower night-time sleep quality. Contrary to previous studies, our study found no significant association between night-time sleep quality and age, sex and medical diagnoses, in particular dementia severity and depression $[2,4,10]$. Both age and dementia are known to adversely impact on circadian rhythms, with increasing dementia severity impacting further on sleep.

There was a statistically significant positive association between regular long-acting benzodiazepine use and higher night-time sleep quality. However, the sample size for regular long-acting benzodiazepines $(n=14)$ was modest in comparison to other categories. Traditionally, long-acting benzodiazepines such as diazepam are more commonly prescribed to manage anxiety disorders [32]. As such, residents using long-acting benzodiazepines may not have had existing sleep problems.

In our study, there was an association between use of short-acting benzodiazepines on a PRN basis only and lower

Table 3 Association between benzodiazepine use and sleep quality, day-time drowsiness and napping in Australian residential aged care facilities

\begin{tabular}{|c|c|c|c|c|c|c|c|c|c|c|}
\hline & \multirow[b]{2}{*}{$N$} & \multicolumn{3}{|c|}{$\begin{array}{l}\text { Association between BZD and higher } \\
\text { night-time sleep quality }\end{array}$} & \multicolumn{3}{|c|}{$\begin{array}{l}\text { Association between BZD and day- } \\
\text { time drowsiness }\end{array}$} & \multicolumn{3}{|c|}{$\begin{array}{l}\text { Association between BZD and longer average } \\
\text { napping time ( } \geq 3.5 \mathrm{~h} \text { per week) }\end{array}$} \\
\hline & & $\mathrm{AOR}^{\mathrm{a}}$ & $\mathrm{Cl}$ & $P$ & $\mathrm{AOR}^{\mathrm{a}}$ & $\mathrm{Cl}$ & $P$ & $\mathrm{AOR}^{\mathrm{a}}$ & $\mathrm{Cl}$ & $P$ \\
\hline Any BZD & 179 & 0.82 & $0.53-1.26$ & 0.36 & 1.27 & $0.81-1.98$ & 0.30 & 1.04 & $0.65-1.66$ & 0.87 \\
\hline \multicolumn{11}{|l|}{ Any Regular } \\
\hline - short-acting & 83 & 0.91 & $0.54-1.53$ & 0.71 & 1.05 & $0.62-1.80$ & 0.85 & 0.70 & $0.40-1.22$ & 0.21 \\
\hline - long-acting & 14 & 4.00 & $1.06-15.15$ & 0.04 & 1.53 & $0.48-4.90$ & 0.48 & 0.32 & $0.07-1.50$ & 0.15 \\
\hline \multicolumn{11}{|l|}{ PRN only } \\
\hline - short-acting & 69 & 0.59 & $0.34-1.02$ & 0.06 & 1.29 & $0.73-2.26$ & 0.38 & 1.77 & $1.01-3.08$ & 0.04 \\
\hline - long-acting & 11 & 0.77 & $0.22-2.67$ & 0.68 & 1.29 & $0.37-4.46$ & 0.69 & 1.18 & $0.30-4.70$ & 0.81 \\
\hline
\end{tabular}

$A O R$ adjusted odds ratio, $C I 95 \%$ confidence interval, BZD benzodiazepine

a each model adjusted for age, sex, DSRS (Dementia Severity Rating Scale), PAINAD (Pain Assessment in Advanced Dementia Scale), depression, insomnia and use of non-benzodiazepine sedatives 
night-time sleep quality, although this was not statistically significant. The effects of benzodiazepine use on sleep quality have been mixed. For example, a systematic review of randomized controlled trials found that sedative hypnotics offer statistically significant, but small, improvements in sleep quality [13]. However, long-term benzodiazepine use is associated with tolerance which may negate the therapeutic effects of benzodiazepines [27]. As our study was crosssectional we were not able to investigate tolerance and duration of benzodiazepine use. A cross-sectional study by Béland et al. also reported an association between benzodiazepines and poor sleep quality, which the authors attributed to the physiological tolerance mechanism [33]. The use of short-acting benzodiazepines, such as temazepam, is recommended as a first line short-term treatment for insomnia by the Australian Therapeutics Guidelines [32]. Thus, this finding may be attributed to the role of using short-acting benzodiazepines, as required, in residents who are experiencing poor sleep quality. Individuals with poor sleep quality may be more likely to be prescribed PRN short-acting benzodiazepines, and this may highlight potential confounding by indication.

There was an association between PRN benzodiazepine only use and longer day-time napping. Australian Therapeutic Guidelines recommend the use of PRN medication in both the treatment of insomnia and anxiety [32]. Historically, short-acting benzodiazepines have been attributed with lower rates of adverse events and lowered risk of residual next-day sequelae such as napping [34]. Clinician awareness of preventing adverse events is seen through prescribing habits and patterns. Thus, our findings may reflect channeling of PRN medications to residents susceptible to daytime napping, particularly those with acute rather than chronic insomnia [35]. This study found no association between benzodiazepine use and day-time drowsiness, whereas previous research indicates benzodiazepine users are at a greater risk of somnolence $[10,28]$. The different effects of varying formulations and regimens of benzodiazepines on the sleep domains highlights the need for informed decision making when it comes to prescribing these medications.

\section{Strengths and limitations}

Demographic and diagnostic characteristics were similar among participants and non-participants, thus the study sample presented is likely representative of all residents in the RACFs. Despite having a larger sample size than previous research [27], generalizability of the study results may be limited and findings may not be applicable to other demographics and settings. The cross-sectional study design limited our ability to infer causality between associated factors.

Strengths lie in our method of data collection. Diagnostic data were extracted from each resident's most recent electronic medical records. Likewise, medication use was extracted from current medication charts, providing a more accurate reflection of actual medication exposure than relying on prescribing or dispensing data to ascertain medication use. We conducted separate analyses for regular and PRN benzodiazepine use and for short and longacting benzodiazepine use. However, we did not investigate the possible impact of benzodiazepine dose and duration, which could have effects through tolerance with long term use, or withdrawal worsening sleep quality in residents with recent dose reduction or recent change from long to short acting benzodiazepines. Consideration was made for a range of clinically relevant covariates; however, we did not adjust our analyses for sleep disorders other than insomnia, such as obstructive sleep apnea and restless leg syndrome [10]. Other potential factors previously studied, such as environment, poor sleep hygiene, social connections and fitness levels, were not considered $[10,20]$. The majority of observational or self-reported data in our study were gathered with widely-used, previously validated scales that have been demonstrated to be appropriate for use in aged care facilities or people with dementia. However, the results should be interpreted in light of the fact that $40.2 \%$ of the sampled residents needed assistance with completing the sleep questionnaire, and a proportion of data were imputed. Residents needing assistance with the questionnaire were largely cognitively impaired. Although a staff or carer proxy was used in these cases, they had to be familiar with the resident and their sleep behavior (i.e. had cared for the resident for a minimum of two weeks). By including residents with cognitive impairment, generalizability within the aged care setting was maximized. The small number of regular long-acting benzodiazepine users, and hence wide confidence interval, means these results should be interpreted with caution.

\section{Conclusions}

The association between benzodiazepine use and sleep quality in residents of aged care facilities is dependent on the half-life and prescribing pattern of the benzodiazepine. Short-acting PRN benzodiazepines were associated with lower night time sleep quality and longer day-time napping compared to long-acting regular benzodiazepines. Longitudinal studies are needed to determine whether these findings reflect channeling of the short-acting agents and PRN prescribing to residents at higher risk of sleep disorders, or acute rather than chronic insomnia. Further research should be undertaken to explore the effect of benzodiazepine dose and duration on sleep quality.

\section{Abbreviations}

AOR: Adjusted odds ratio; ATC: Anatomical Therapeutic Classification;

$\mathrm{Cl}$ : Confidence interval; GABA: Gamma-Aminobutyric acid; OR: Odds ratio;

PRN: Pro re nata; RACF: Residential aged care facility; RACGP: Royal Australian

College of General Practitioners; REM: Rapid eye movement 


\section{Acknowledgements}

This research was funded by the Alzheimer's Australia Dementia Research Foundation via the Resthaven Incorporated Dementia Research Award, with additional funding provided by Resthaven Incorporated. ECKT was supported by a NHMRC-ARC Dementia Research Development Fellowship. The authors would like to thank Resthaven staff and residents for their participation and support in this study. We thank Mary Jones, Sarah Moawad and Jenna Huskic for their assistance with data management.

\section{Funding}

This research was funded by the Alzheimer's Australia Dementia Research Foundation via the Resthaven Incorporated Dementia Research Award, with additional funding provided by Resthaven Incorporated. ECKT was supported by a NHMRC-ARC Dementia Research Development Fellowship.

\section{Availability of data and materials}

The dataset is available on request by the authors.

\section{Authors' contributions}

JSB, RV, SNH and AIV conceived the study and obtained grant funding. JSB, ECKT, RV and SNH participated in the study design. TE and LR assisted in the development and implementation of the study. Data were managed by $\mathrm{JMH}$ and ECKT. LC and ECKT performed the data analysis. LC and ECKT drafted the manuscript. All authors read and approved the final manuscript.

\section{Competing interests}

The authors declare that they have no competing interest.

\section{Consent to publish}

Not applicable.

\section{Ethics approval and consent to participate}

The study was approved by the Royal Australian College of General Practitioners (RACGP) National Research and Evaluation Ethics Committee and the Monash University Human Research Ethics Committee. All participants were informed about the study verbally and in writing. Written informed consent was obtained from the residents, or their guardian, next of kin, or significant other if unable. All study procedures were conducted in accordance with the Australian National Statement for Ethical Conduct in Human Research.

\section{Author details}

${ }^{1}$ Faculty of Medicine, Nursing and Health Sciences, Monash University, Melbourne, Australia. ${ }^{2}$ Centre for Medicine Use and Safety, Faculty of Pharmacy and Pharmaceutical Sciences, Monash University, 381 Royal Parade, Melbourne, Parkville Victoria 3052, Australia. ${ }^{3}$ NHMRC Cognitive Decline Partnership Centre, Hornsby Ku-ring-gai Hospital, Hornsby, Australia. ${ }^{4}$ Sansom Institute, School of Pharmacy and Medical Sciences, University of South Australia, Adelaide, Australia. ${ }^{5}$ Discipline of Medicine, University of Adelaide, Adelaide, Australia. ${ }^{6}$ Aged and Extended Care Services, The Queen Elizabeth Hospital and Adelaide Geriatrics Training and Research with Aged Care (GTRAC) Centre, School of Medicine, University of Adelaide, Adelaide, Australia. ${ }^{7}$ Kolling Institute of Medical Research, Departments of Clinical Pharmacology and Aged Care, Royal North Shore Hospital, Sydney Medical School, The University of Sydney, Sydney, Australia. ${ }^{8}$ Resthaven Limited, Adelaide, Australia. ${ }^{9}$ Aging Research Center, Center for Alzheimer Research, Department of Neurobiology, Care Sciences and Society, Karolinska Institutet and Stockholm University, Stockholm, Sweden.

Received: 17 May 2016 Accepted: 13 November 2016

Published online: 26 November 2016

\section{References}

1. Rajput V, Bromley SM. Chronic insomnia: a practical review. Am Fam Physician. 1999;60(5):1431-8.

2. Wolkove N, Elkholy O, Baltzan M, Palayew M. Sleep and aging: 1. Sleep disorders commonly found in older people. CMAJ. 2007;176(9):1299-304.

3. Bloom HG, Ahmed I, Alessi CA, Ancoli-lsrael S, Buysse DJ, Kryger MH, Phillips BA, Thorpy MJ, Vitiello MV, Zee PC. Evidence-based recommendations for the assessment and management of sleep disorders in older persons. J Am Geriatr Soc. 2009;57(5):761-89.
4. Monane M. Insomnia in the elderly. J Clin Psychiatry. 1992;53(Suppl):23-8.

5. Gindin J, Shochat T, Chetrit A, Epstein S, Ben Israel Y, Levi S, Onder G, Carpenter I, Finne-Soveri $\mathrm{H}$, van Hout $\mathrm{H}$, et al. Insomnia in long-term care facilities: a comparison of seven European countries and Israel: the Services and Health for Elderly in Long TERm care study. J Am Geriatr Soc. 2014; 62(11):2033-9.

6. Australian Institute of Health and Welfare. Residential aged care and Home Care 2013-14. 2014. http://www.aihw.gov.au/aged-care/residential-andhome-care-2013-14/\#toc. Accessed 27 July 2016.

7. Rongve A, Boeve BF, Aarsland D. Frequency and correlates of caregiverreported sleep disturbances in a sample of persons with early dementia. J Am Geriatr Soc. 2010;58(3):480-6.

8. Tan ECK, Jokanovic N, Koponen MP, Thomas D, Hilmer SN, Bell JS Prevalence of analgesic use and pain in people with and without dementia or cognitive impairment in aged care facilities: a systematic review and meta-analysis. Curr Clin Pharmacol. 2015;10(3):194-203.

9. Ouslander JG, Connell BR, Bliwise DL, Endeshaw Y, Griffiths P, Schnelle JF. A nonpharmacological intervention to improve sleep in nursing home patients: results of a controlled clinical trial. J Am Geriatr Soc. 2006;54(1):38-47.

10. Conn DK, Madan R. Use of sleep-promoting medications in nursing home residents : risks versus benefits. Drugs Aging. 2006;23(4):271-87.

11. Westbury JL, Jackson S, Peterson GM. Psycholeptic use in aged care homes in Tasmania, Australia. J Clin Pharm Ther. 2010;35(2):189-93.

12. Holbrook A, Crowther R, Lotter A, Endeshaw Y. The role of benzodiazepines in the treatment of insomnia: meta-analysis of benzodiazepine use in the treatment of insomnia. J Am Geriatr Soc. 2001;49(6):824-6.

13. Glass J, Lanctot KL, Herrmann N, Sproule BA, Busto UE. Sedative hypnotics in older people with insomnia: meta-analysis of risks and benefits. BMJ. 2005;331(7526):1169.

14. Tampi RR, Tampi DJ. Efficacy and tolerability of benzodiazepines for the treatment of behavioral and psychological symptoms of dementia: a systematic review of randomized controlled trials. Am J Alzheimers Dis Other Demen. 2014;29(7):565-74.

15. Puustinen J, Nurminen J, Vahlberg T, Lyles A, Isoaho R, Raiha I, Kivela SL. CNS medications as predictors of precipitous cognitive decline in the cognitively disabled aged: a longitudinal population-based study. Dement Geriatr Cogn Dis Extra. 2012;2(1):57-68.

16. McCleery J, Cohen DA, Sharpley AL. Pharmacotherapies for sleep disturbances in Alzheimer's disease. Cochrane Database Syst Rev. 2014;3: CD009178.

17. Zammit GK, Weiner J, Damato N, Sillup GP, McMillan CA. Quality of life in people with insomnia. Sleep. 1999;22 Suppl 2:S379-385.

18. Tan ECK, Visvanathan R, Hilmer SN, Vitry Al, Quirke T, Emery T, Robson L, Shortt $\mathrm{T}$, Sheldrick S, Lee SS, et al. Analgesic use, pain and daytime sedation in people with and without dementia in aged care facilities: a cross-sectional, multisite, epidemiological study protocol. BMJ Open. 2014;4(6):e005757.

19. Hayward LB, Mant A, Eyland EA, Hewitt H, Pond CD, Saunders NA. Neuropsychological functioning and sleep patterns in the elderly. Med J Aust. 1992;157(1):51-2

20. St George RJ, Delbaere K, Williams P, Lord SR. Sleep Quality and Falls in Older People Living in Self- and Assisted-Care Villages. Gerontology. 2009; 55(2):162-8

21. Charlson ME, Pompei P, Ales KL, MacKenzie CR. A new method of classifying prognostic comorbidity in longitudinal studies: development and validation. J Chronic Dis. 1987:40(5):373-83.

22. Clark CM, Ewbank DC. Performance of the dementia severity rating scale: a caregiver questionnaire for rating severity in Alzheimer disease. Alzheimer Dis Assoc Disord. 1996;10(1):31-9.

23. Wood S, Cummings JL, Hsu MA, Barclay T, Wheatley MV, Yarema KT, Schnelle JF. The use of the neuropsychiatric inventory in nursing home residents. Characterization and measurement. Am J Geriatr Psychiatry. 2000; 8(1):75-83.

24. Katz S, Ford AB, Moskowitz RW, Jackson BA, Jaffe MW. Studies of illness in the aged. The index of ADL: a standardized measure of biological and psychosocial function. JAMA. 1963;185:914-9.

25. Warden V, Hurley AC, Volicer L. Development and psychometric evaluation of the Pain Assessment in Advanced Dementia (PAINAD) scale. J Am Med Dir Assoc. 2003:4(1):9-15.

26. Janssen KJ, Donders AR, Harrell Jr FE, Vergouwe Y, Chen Q, Grobbee DE, Moons KG. Missing covariate data in medical research: to impute is better than to ignore. J Clin Epidemiol. 2010;63(7):721-7. 
27. Bourgeois J, Elseviers MM, Bortel LV, Petrovic M, Vander Stichele RH. Sleep quality of benzodiazepine users in nursing homes: A comparative study with nonusers. Sleep Med. 2013;14(7):614-21.

28. de Vries OJ, Peeters G, Elders P, Sonnenberg C, Muller M, Deeg DJH, Lips P. The elimination half-life of benzodiazepines and fall risk: two prospective observational studies. Age Ageing. 2013;42(6):764-70.

29. Westbury JL, Peterson GM. Rethinking psychotropics in nursing homes. Med J Aust. 2013;199(2):98.

30. Bourgeois J, Elseviers MM, Azermai M, Van Bortel L, Petrovic M, Vander Stichele RR. Benzodiazepine use in Belgian nursing homes: a closer look into indications and dosages. Eur J Clin Pharmacol. 2012;68(5):833-44.

31. Lee DR, Thomas AJ. Sleep in dementia and caregiving-assessment and treatment implications: a review. Int Psychogeriatr. 2011;23(2):190-201.

32. Therapeutic Guidelines Limited. eTG complete. Melbourne: Therapeutic Guidelines Limited; 2014

33. Béland S-G, Préville M, Dubois M-F, Lorrain D, Grenier S, Voyer P, Pérodeau $G$, Moride Y. Benzodiazepine use and quality of sleep in the communitydwelling elderly population. Aging Ment Health. 2010;14(7):843-50.

34. Nicholson AN. The use of short- and long-acting hypnotics in clinical medicine. Br J Clin Pharmacol. 1981;11(S1):61S-9S.

35. Woodward MC. Managing insomnia in older people. J Pharm Pract Res. 2007:37:236-41.

\section{Submit your next manuscript to BioMed Central} and we will help you at every step:

- We accept pre-submission inquiries

- Our selector tool helps you to find the most relevant journal

- We provide round the clock customer support

- Convenient online submission

- Thorough peer review

- Inclusion in PubMed and all major indexing services

- Maximum visibility for your research

Submit your manuscript at www.biomedcentral.com/submit 